\title{
Sleep Disturbances in Patients with Nonepileptic Seizures
}

This article was published in the following Dove Press journal:

Nature and Science of Sleep

\author{
Jakub Vanek (D) \\ Jan Prasko (iD ${ }^{1-3}$ \\ Marie Ociskova (D) \\ Samuel Genzor (iD ${ }^{4}$ \\ Michaela Holubova ${ }^{5}$ \\ Frantisek Hodny (D) \\ Vlastmil Nesnidal (D) \\ Milos Slepecky ${ }^{3}$ \\ Milan Sova $\mathbb{D D}^{4}$ \\ Kamila Minarikova (D) \\ 'Department of Psychiatry, Faculty of \\ Medicine and Dentistry, University \\ Hospital, University Palacky Olomouc, \\ Olomouc, 77520, The Czech Republic; \\ ${ }^{2}$ Institute for Postgraduate Education in \\ Health Care, Prague, The Czech \\ Republic; ${ }^{3}$ Department of Psychology \\ Sciences, Faculty of Social Science and \\ Health Care, Constantine the \\ Philosopher University in Nitra, Nitra, \\ The Slovak Republic; ${ }^{4}$ Department of \\ Respiratory Medicine, University \\ Hospital Olomouc and Faculty of \\ Medicine and Dentistry, Palacky \\ University Olomouc, Olomouc, The \\ Czech Republic; ${ }^{5}$ Department of \\ Psychiatry, Hospital Liberec, Liberec, The \\ Czech Republic
}

Correspondence: Jan Prasko Department of Psychiatry, Faculty of Medicine and Dentistry, University Hospital, University Palacky Olomouc, I. P. Pavlova 6, Olomouc, 77900, The

Czech Republic

Email praskojan@seznam.cz
Objective: Up to $20 \%$ of patients treated for epileptic seizures experience psychogenic nonepileptic paroxysms (PNES). These patients present a significant burden for the health care systems because of poor treatment outcomes. The presented review aims to summarize the current state of knowledge on sleep disturbances in patients with nonepileptic seizures. Methods: Articles were acquired via PubMed and Web of Science, and papers between January 1990 and March 2020 were extracted. Inclusion criteria were (1) published in a peerreviewed journal: (2) studies in humans only; or (3) reviews on a related topic; (4) English language. The exclusion criteria were: (1) abstracts from conferences; (2) commentaries; (3) subjects younger than 18 years. From primary assessment, 122 articles were extracted; after obtaining full texts and secondary articles from reference lists, 45 papers were used in this review.

Results: Limited data are available regarding sleep disorders in PNES patients, over the last 30 years only nine original research papers addressed sleep problems in patients with PNES with only six studies assessing objectively measured changes in sleep. Current literature supports the subjective perception of the sleep disturbances with mixed results in objective pathophysiological findings. Conflicting results regarding the REM phase can be found, and studies reported both shortening and prolonging of the REM phase with methodological limitations. Poor sleep quality and shortened duration have been consistently described in most of the studies.

Conclusion: Further research on a broader spectrum of patients with PNES is needed, primarily focusing on objective neurophysiological findings. Quality of life in patients suffering from PNES can be increased by good sleep habits and treatment of comorbid sleep disorders.

Keywords: psychogenic seizures, sleep disturbances, insomnia, mental disorders, emotional regulation

\section{Introduction}

As many as one-fifth of patients, who show up at specialized neurological departments with seizures do not meet the diagnostic criteria for epilepsy. Most of these patients suffer from dissociative seizures, otherwise known as "nonepileptic seizures". These dissociative seizures (otherwise psychogenic nonepileptic seizures, PNES) are, according to DSM-5, a type of the conversion disorder belonging to the functional neurological symptom disorders (FNSD). ${ }^{1}$ They manifest with paroxysmal, sudden changes in motor activity, behaviour, cognitive processing with changes in consciousness, or autonomic functions associated with dysfunction in the processing of anxiety. ${ }^{2-4}$ PNES episodes may include motor behaviour, intrinsic 
sensory or cognitive deviations. PNES can be confused with partial or generalized epileptic seizures, and the patient may continue to communicate with the environment or stop responding. ${ }^{5}$ PNES is a complex neuropsychiatric disorder at the border of neurological and psychiatric disciplines that has been largely overlooked and avoided by mental health providers. ${ }^{6-8}$ The diagnosis of PNES is also made in 20 to $50 \%$ of inpatients in departments specialized in epilepsy ${ }^{9,10}$ and can be present in up to $25 \%$ of patients treated for refractory epilepsy. ${ }^{11-14}$ The prevalence estimates are further complicated by common comorbidity between PNES and epilepsy that occurs in $20 \%$ to $60 \%$ of the individuals with epilepsy. ${ }^{15}$ PNES are more prevalent in women, most of whom are aged between 15 and 35 years. ${ }^{8,16,17}$

Patients with PNES have high health care consumption, and they are reaching a level of disability in everyday life similar to that of patients with epilepsy. ${ }^{18,19}$ Their prospective treatment outcomes are not favourable. In a 10-year follow-up study, the treatment outcome was rated as poor in $44 \%$ of patients (seizures persisted or patients remained dependent on care). ${ }^{20}$

Despite advances in diagnostic and evidence-based treatment, current knowledge about PNES has not been sufficiently translated into clinical practice. ${ }^{8,21}$ In some studies, the diagnosis of PNES is delayed up to nine years after the onset of symptoms. ${ }^{21,22}$ The delayed diagnosis is then associated with poor prognosis. ${ }^{22-24}$ Although the diagnosis should be made by video electroencephalograph (vEEG), most health care providers do not use this test. ${ }^{24}$ Unfortunately, this can lead to an unnecessary overlook of PNES diagnosis as vEEG usually detects seizures that do not have EEG correlates of the epileptic activity. However, there are also situations where even long-term follow-up does not lead to the recording of typical seizures. $^{25}$

Also, the pathophysiology of PNES is not well understood. $^{26,27}$ Physical or sexual abuse in anamnesis is frequent. It occurs in up to $84 \%$ of these patients. ${ }^{27-29}$ Patients with PNES are characterized by difficulty verbalizing their emotions, especially when anxious and manifest them via somatic symptoms instead. ${ }^{30}$ Recent advances in the search for possible neurophysiological biomarkers in functional MRI studies indicate abnormalities in emotional, cognitive, executive, and sensorimotor neuronal circuits. ${ }^{24,31,32}$ Psychobiological studies have shown that patients with PNES may be less tolerant of emotional arousal ${ }^{33}$ and show high alexithymia (difficulty identifying and describing internal emotional experiences), ${ }^{3,34,35}$ dissociation, ${ }^{6,36}$ somatization rates, ${ }^{3}$ and tendencies to avoid emotions altogether. ${ }^{36,37}$ Some researchers describe patients with PNES is a heterogeneous group. ${ }^{26,31,38,39}$ However, KalogjeraSackellares (1996) described two leading "causes" of PNES: post-traumatic PNES, acute or distant trauma, and developmental PNES emotional deprivation or psychological trauma during developmental periods. ${ }^{40}$ As noted above, PTSD and history of traumatic events are highly prevalent among patients with PNES..$^{27,41,42}$ Some authors have conceptualized PNES as manifestations of PTSD; ${ }^{43}$ however, only half of the patients with PNES have PTSD. ${ }^{28}$ Several comorbidities are usually present, especially mood, anxiety and sleep disorders, personality disorders, chronic pain other disorders with somatic symptoms. $^{44}$

Patients with PNES are prone to more somatic symptoms than the healthy control group or patients with epilepsy. Examples of such symptoms are the higher incidence of somatic syndromes (fibromyalgia, chronic pain, chronic fatigue syndrome, migraine headaches), ${ }^{45}$ mood and anxiety disorders (including PTSD) and personality disorders. ${ }^{41,42,46}$ These comorbid conditions can and should be addressed together with dissociative seizures in complex neuropsychiatric treatment. Regarding treatment, various psychotherapies and pharmacological therapies for PNES have been investigated. ${ }^{7,24,26}$ The treatment of choice is cognitive behavioural therapy. ${ }^{42,47,48}$

According to literature maintaining proper sleep hygiene and the daily regime is essential in managing patients with PNES and it is discussed whether sleep disorders can have an effect and if so to what extent they can disrupt treatment of patients with PNES. ${ }^{44}$ Our first focus was to objectify whether PNES can occur in sleep according to contemporary literature and researches and whether there are specific changes in sleep patterns that can be quantified. Since insomnia and changes in the sleep duration, in general, are associated with various psychiatric disorders, we tried to establish whether there is a link between sleep duration and dissociative seizures. Clinical praxis needs to understand specific sleep problems in patients with PNES to improve the quality of life and address these issues in the management and treatment plan. Following research questions were formulated:

(1) Do dissociative episodes occur during the sleep or only while awake? 
(2) Does the total length of sleep differ from the norm in patients with dissociative seizures? Are there any objectively assessed changes in sleep duration?

(3) Is the sleep architecture altered in patients with dissociative episodes?

(4) Do patients with dissociative seizures experience sleep disorders other than insomnia?

\section{Method}

These narrative review articles were obtained via PubMed and Web of Science, and papers between January 1990 and March 2020 were extracted. We made a sequence of literature searches using the keywords: dissociative seizures, sleep disorders, insomnia, PNES and psychogenic nonepileptic paroxysms. Keywords were used in all possible permutations. Selected papers met inclusion criteria: (1) published in peer-reviewed journals; (2) human studies; or (3) reviews on the related topic; (4) English language. We included original articles that diagnosed patients via vEEG according to DSM-5 or ICD-10 criteria. Articles included both patients with exclusive PNES a patient with comorbid epilepsy. The exclusion criteria were: (1) abstracts from conferences; (2) commentaries; (3) subjects younger than 18 years. We excluded studies in children, reasoning the neurodevelopmental nature of sleep in them. More articles were found from the references of the primary articles. The primary process selected a total of 122 items. Adjusting for the inclusion and exclusion criteria, 34 pieces were selected. After a full-text assessment by the research team, 21 papers were included, all articles and

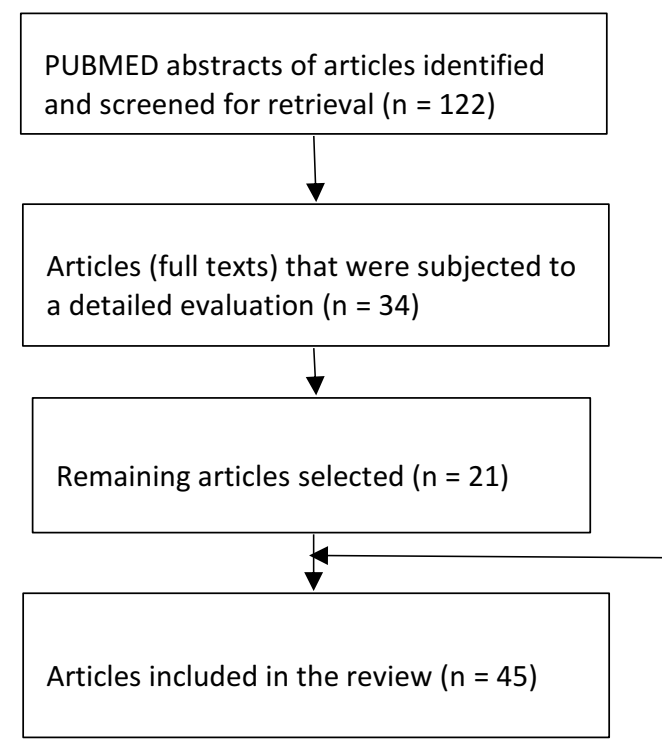

their inclusion were subjected to open discussion and decision by the research team. Secondary papers from the primary articles' references were also searched, and eligible papers were added to the first list $(\mathrm{n}=24)$. In summary, 45 papers were included in the review (Figure 1). ${ }^{49}$

\section{Results}

Contemporary literature included in this review found predominantly subjective changes in sleep in patients with PNES. There is a lack of objective evidence to support these subjective complaints. ${ }^{50}$ According to one study, patients with PNES, are more prone to sleep disturbances than patients with epilepsy. ${ }^{51}$ We further divide our findings according to the research questions and summarise the table of original research papers included in this review (Table 1).

\section{Do Dissociative Seizures Occur During Sleep or Only While Awake?}

Patients with PNES frequently describe dissociative seizures occurring in sleep, when monitored by telemetry they are usually preceded by awakening, ${ }^{52,53}$ which can be recorded on EEG. It is an open question of whether dissociative seizures may occur in sleep or not.

One of the first studies by Thacker et al reported dissociative seizures in 12 patients, who seemed to be asleep, but according to video-EEG, were awake. ${ }^{52}$ Later works reported up to $13 \%$ of PNES episodes happens
Secondary search articles from the reference lists of the remaining articles after a detailed evaluation $(n=24)$

Figure I Summary of the selection process. 


\begin{tabular}{|c|c|c|c|c|c|c|c|c|}
\hline $\begin{array}{l}\frac{\tilde{y}}{\vec{J}} \\
\bar{y} \\
\ddot{\varphi}\end{array}$ & 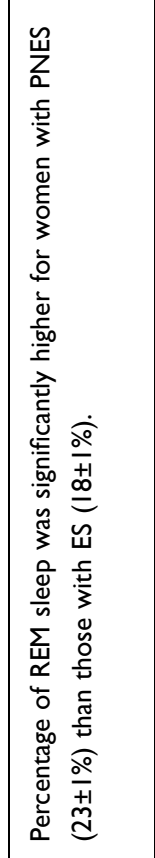 & 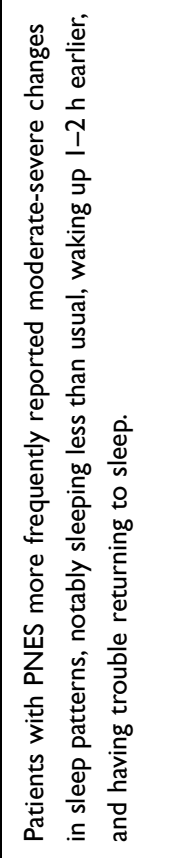 & 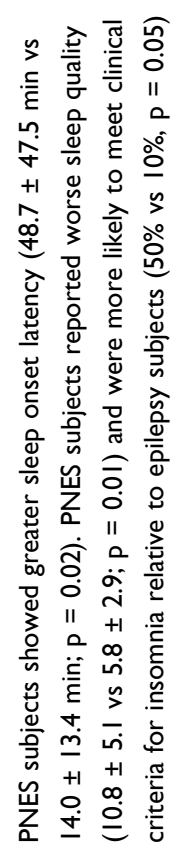 & 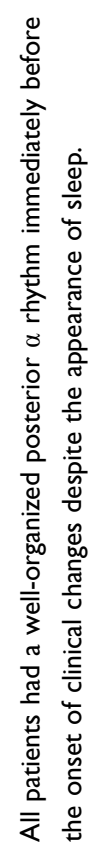 & 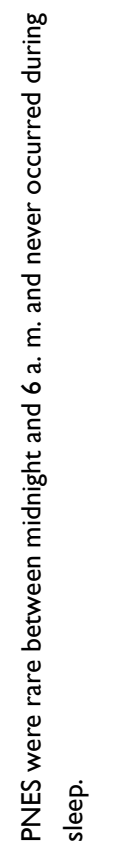 & 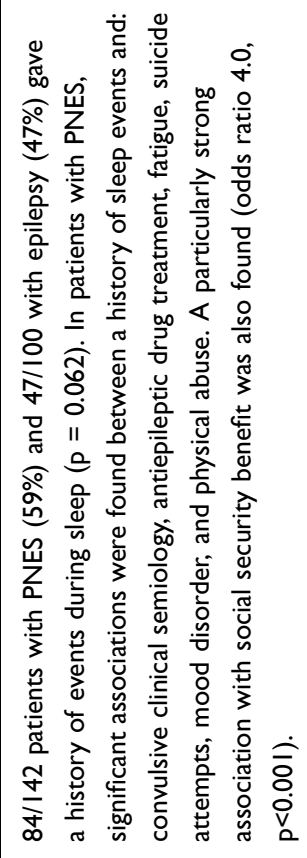 & 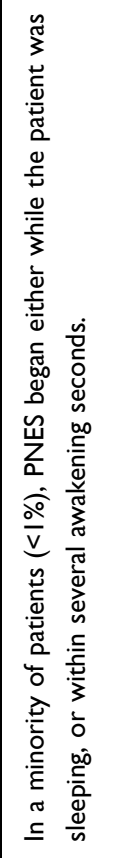 & 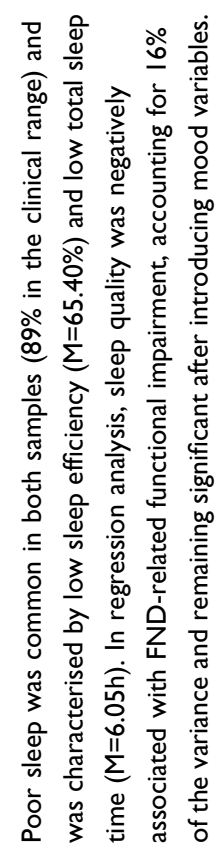 \\
\hline 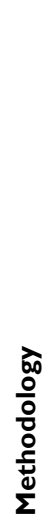 & 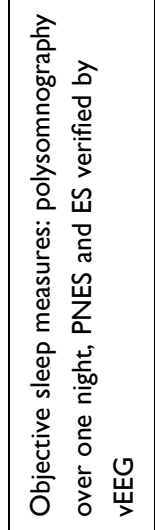 & 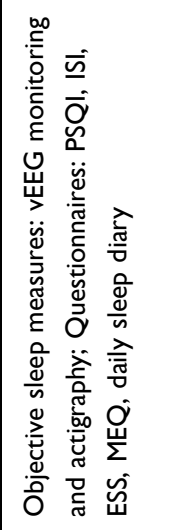 & 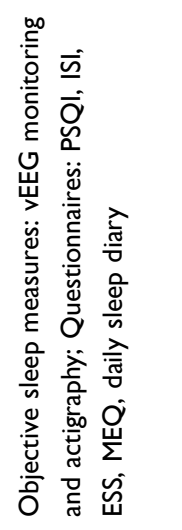 & 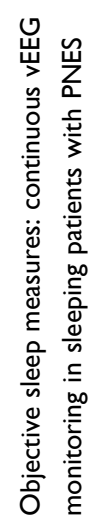 & 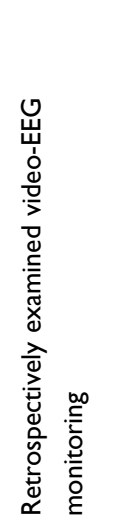 & 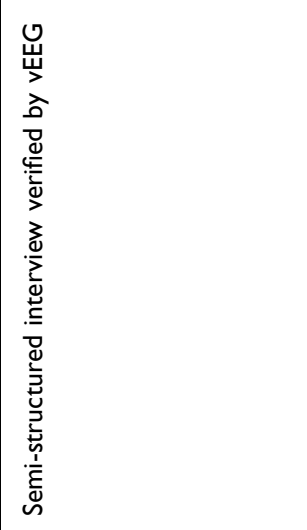 & 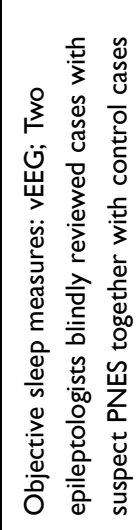 & 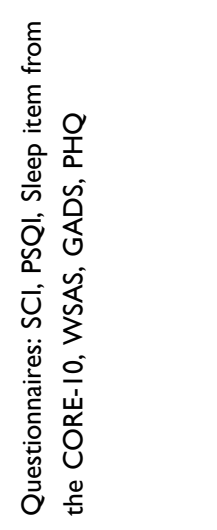 \\
\hline 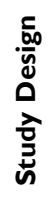 & 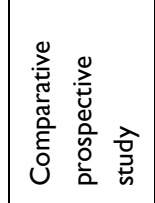 & 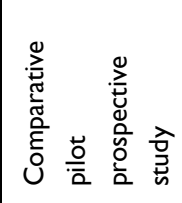 & 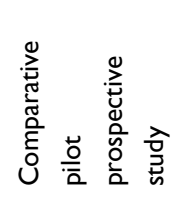 & 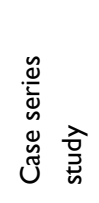 & 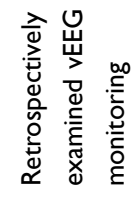 & 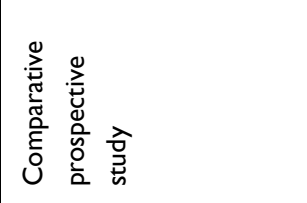 & 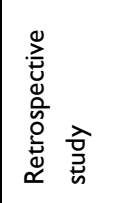 & 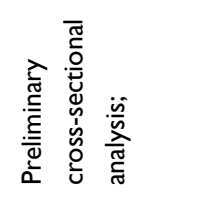 \\
\hline $\begin{array}{l}\frac{N}{n} \\
\frac{\tilde{n}}{0} \\
\frac{0}{\tilde{n}} \\
\text { ñ }\end{array}$ & 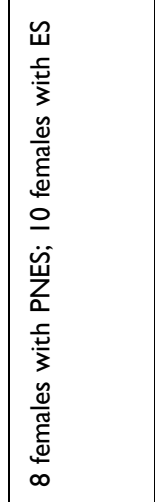 & 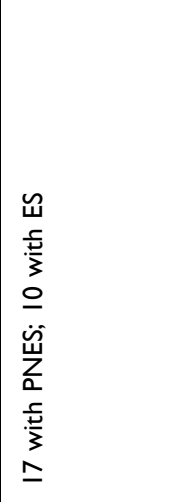 & 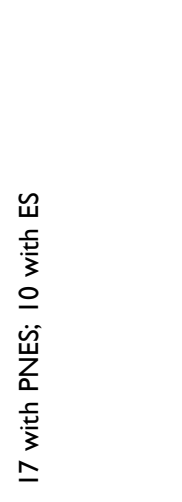 & 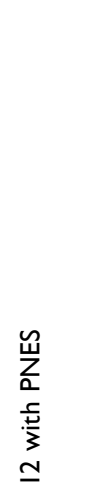 & 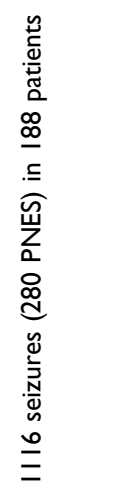 & 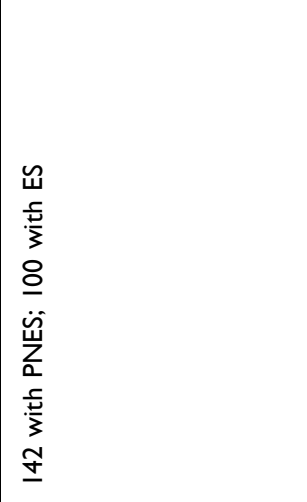 & $\begin{array}{l}\frac{\tilde{u}}{0} \\
\frac{.0}{0} \\
\bar{z} \\
\hat{N}\end{array}$ & 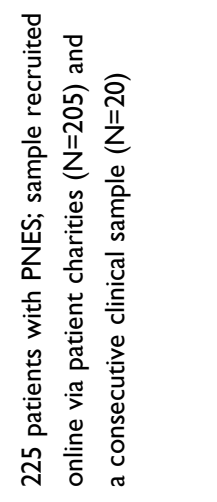 \\
\hline $\bar{J}$ & 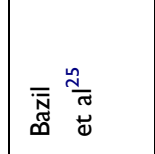 & 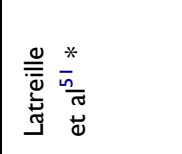 & 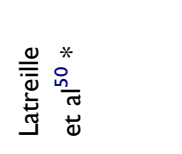 & 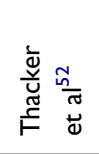 & $\frac{n}{\infty}$ & 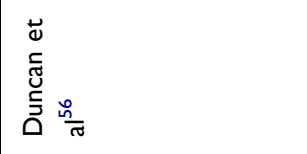 & 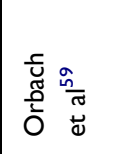 & 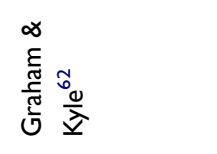 \\
\hline
\end{tabular}




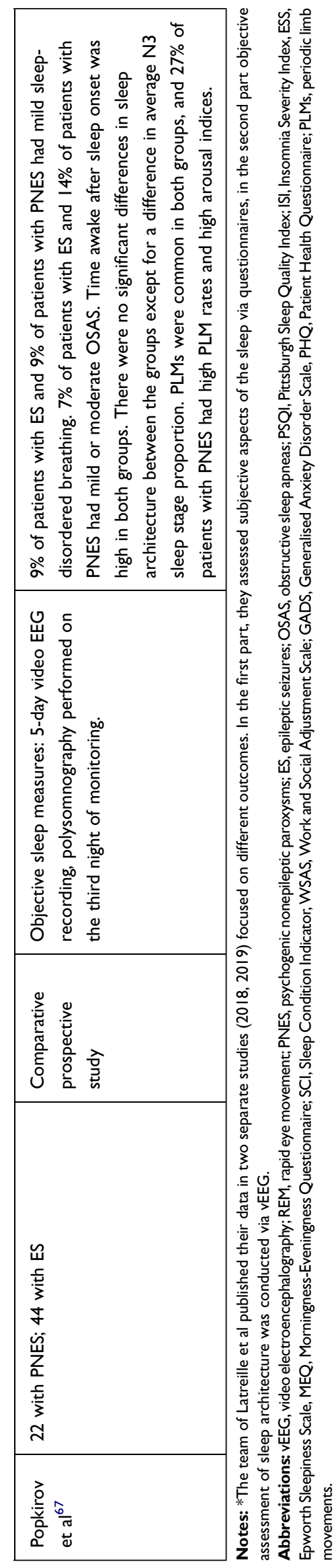

during seeming sleep. ${ }^{5}$ However, dissociative convulsions have rarely been observed in proper sleep confirmed by electroencephalography (EEG). ${ }^{54}$

PNES are not evenly dispersed throughout 24 hours. Psychogenic symptoms are believed to be only present when the person is awake. If PNES happens during sleep, studies imply that they are awake with their closed eyes (experiences "a pseudo-sleep"). In contrast, epileptic seizures often occur during normal sleep. ${ }^{52,55,57,58}$ Still, Orbach et al found that a small proportion of patients $(<1 \%)$ can develop PNES during the sleep (most commonly in N2 or N3 of NREM (non-rapid eye movement sleep) or within seconds of waking up. The EEG finding of the seizure itself was at the waking stage. ${ }^{59}$ Similarly, Seneviratne et al found that the chances of developing PNES in wakefulness are approximately four times higher compared with the sleeping state (ODDs-ratio: 4.27, 95\% CI: 2.44-7.48). Moreover, he found that the majority of dissociative seizures occurred in the night interval while being awake. ${ }^{60}$

There is also another quite common research problem in patients who experience dissociative seizures, it is unlikely that the episode will occur when monitored. Patients with different types of paroxysms also require a different approach. Both dissociative seizures and epileptic seizures may be present in the same patient. Besides, a dissociative attack in a patient with epilepsy can appear on the telemetry unit, perhaps from the stress caused by the pressure to "perform" and have the attack recorded. ${ }^{61}$

In summary, PNES onsets are much more frequent in an awake state, most commonly in the night interval. Even though patients with PNES states that they are asleep at the onset of the dissociative seizure, objective methods suggest that PNES event is preceded by awakening and true nature of dissociative seizures in sleep is still unclear.

\section{Does the Subjective Sleep Length Differ from the Norm in Patients with Dissociative Seizures? Are There Objectively Assess Changes in Sleep Duration?}

Phillips et al showed that patients with PNES reported worse subjective sleep quality than epilepsy patients and a control patient group. The frequency of clinically diagnosed sleep disorders in PNES patients (29\%) did not diverge from patients with epilepsy $(21 \%)$ and the control group $(22 \%)$. Those differences were not explainable by 
different medication, BMI of the patients or comorbid sleep-disordered breathing. ${ }^{13}$ In a study with a larger group of patients with probable neurological disorders, counting PNES as well, up to $89 \%$ of the sample were at high risk group for the insomnia diagnosis. ${ }^{62}$ Patients in the study also presented subjectively worse quality of sleep, reduced sleep time compared to the healthy sample, and those were negatively connected with functional impairment in everyday functioning. ${ }^{62}$

Latreille et al recently pointed out that PNES patients are more prone to poor sleep hygiene than patients with epilepsy. The paper further compared sleep problems in patients with PNES and patients with epilepsy. Video electroencephalography (vEEG) was performed and validated subsequently by a neurologist - the subjects of the study filled in the Beck Depression Inventory-II (BDI-II). One item of the said inventory is focused on the changes in the sleep patterns. All subjects also filled in the Epilepsy Quality of Life Inventory-10 (QOLIE-10), which assesses health-related aspects of life quality in epilepsy patients. $^{50,63}$ This tool was initially validated for use in epilepsy, nowadays it is used in PNES patients. ${ }^{64}$

Compared to the epilepsy group, patients with PNES were more likely to have mild to severe sleep changes, mostly shorter sleep length by up to 1-2 hours, and difficulty falling asleep again. These changes in sleep patterns were linked with lower quality of life. These results propose that sleep disturbance is a more pronounced problem in PNES than in epilepsy. It is not identified whether it can be attributed to paradoxical insomnia (or "poor perception of sleep", a tendency to underestimate total sleep time) or whether PNES patients are more prone to sleep problems than a healthy population. They may also be at risk due to overlapping somatic and psychiatric diagnoses. ${ }^{64}$ The cited paper says that sleep disorders can be more prevalent among PNES patients, up to one-third of the studied group had been diagnosed with the sleep disorder. If we manage to improve sleep, we can observe a decrease in various symptoms of PNES, and that can lead to improvement of the patient's quality of life and further alleviate other symptoms of PNES. A recent study reported that comorbid sleep disorders in patients with mental illnesses could harm treatment results and serve as barriers to treatment. $^{65}$

In summary, only limited data regarding objective changes in sleep duration are available in the current literature. Over 30 years included in our review, we found only three research papers that worked explicitly with sleep duration in patients with PNES and proposed that sleep disturbance can significantly affect life quality. However, the research papers did not confirm subjective complaints via polysomnography. It is advisable to look for sleep disorders in PNES patients and treat them if possible, for future research, we propose an objective assessment of sleep via polysomnography on a larger sample of patients with PNES.

\section{Is the Sleep Architecture Altered in Patients with Dissociative Seizures?}

One study examined sleep architecture in eight PNES subjects and ten epilepsy patients via EEG in specialized epilepsy department. ${ }^{25}$ The authors examined the structure of sleep by polysomnography, comparing PNES and epilepsy patients. Video-EEG monitoring validated by neurologists verified the epileptic seizures and PNES, and clinical neurophysiologists and psychiatrist evaluated patients with PNES. During the study, the patients did not have any anticonvulsants or antidepressants. Caffeine consumption was prohibited, and all patients with diagnosed sleep disorders were excluded, although this exclusion was made solely on anamnestic data. During polysomnography and 24 hours, long observation after no seizures were captured that would disrupt the sleep structure. ${ }^{66}$ Study found that the proportion of REM sleep was more notable in females PNES patients (23\%) than in epileptic female patients (18\%). REM latency was slightly lower in patients with PNES, although this difference was not statistically significant. No significant differences were found in the sleep duration, slow-wave sleep or sleep efficiency. Besides, there were no differences in N1 and N2 stages. This paper proposes that PNES patients have similar architecture disturbances to patients with major depression with increased REM sleep. ${ }^{66}$

Two limited studies $(\mathrm{n}<20)$ reported worse sleep quality reported by patients ${ }^{13}$ and prolonged REM phases during polysomnography in patients with PNES than epilepsy patients. $^{25}$

Regarding the objective sleep architecture, the authors of the study Latreille et al found a nonsignificant trend towards a reduced duration of the REM phase in PNES patients compared to individuals with epilepsy. ${ }^{50}$ This contradicts the Bazil et al results, which found the opposite relationship. This difference may be caused by the discontinuation of antidepressants in Bazil et al study. ${ }^{66}$ One 
possible exploration of the REM phase increase in PNES patients is a rebound phenomenon.

Two recent studies by Popkirov et al and Latreille et al found that sleep architecture was generally comparable in patients with PNES and epilepsy. ${ }^{51,67}$ Popkirov et al examined the occurrence of sleep disorders in a group of 22 PNES patients and 44 epilepsy patients. ${ }^{67}$ In contrast to Bazil et al findings, no differentiation in the REM phase was observed; instead, patients with PNES had shorter slow-wave sleep duration than patients with epilepsy. ${ }^{66}$

Latreille et al compared sleep-wake patterns in a prospective observational study in PNES and epilepsy patients. Twenty-seven subjects were included in the study, of which 17 had PNES, and 10 were diagnosed epilepsy. Compared to epilepsy controls, the PNES patients showed increased latency of sleep onset (on average about 30 minutes longer than controls). Otherwise, both groups had a similar sleep architecture. However, the PNES patients subjectively had poorer sleep quality measured by Pittsburgh Sleep Quality Index $(10.8 \pm 5.1$ versus 5.8 $\pm 2.9 ; \mathrm{p}=0.01$; higher score indicates the worse quality of sleep) and met the clinical criteria for insomnia more often than patients with epilepsy ( $50 \%$ vs $10 \%, \mathrm{p}=0.05)$. In addition, a higher number of patients with PNES admits taking sleep medications $(44 \%$ vs $0 \%, \mathrm{p}=0.01) .{ }^{51} \mathrm{In}$ general, the authors found more evidence about subjective foundation rather than an objective basis of sleep disturbances in patients with PNES. Patients with PNES are more likely to report generally worse sleep quality and almost $70 \%$ report usage medication for sleep. The majority of patients with PNES met the diagnostic criteria for moderate to severe insomnia. However, actigraphy did not show this trend; both groups had similar sleep latency and showed comparable motor activity during the night and day. It can be theorised that based on the fact that the sensor measures motor activity, sleep latency may be underestimated when patients are at rest or even when they are awake. Otherwise, the sleep architecture between PNES and epilepsy was very similar. ${ }^{51}$

In summary, these studies imply that sleep disorders are common in PNES patients. Still, there is no consistent objective confirmation of sleep structure changes in this group than patients with epilepsy. Besides, it appears that in patients with PNES, sleep disorders may be associated with an increased tendency to somatisation, another form of conversion symptom associated with PNES and other functional disorders. ${ }^{7}$

\section{Do Patients with Dissociative Attacks Have Sleep Disorders Other Than Insomnia?}

Sleep can be useful in distinguishing PNES from other neurological disorders. Pseudo-cataplexy ("psychogenic" narcolepsy), "pseudo-parasomnia" and PNES may have a similar appearance. ${ }^{58}$ Cataplexy and parasomnia are the most common sleep disorders that can be confused with epilepsy. ${ }^{68,69}$ Cataplexy is a sudden loss of muscle tone or falls caused by strong positive or negative emotions; it is usually a symptom of narcolepsy. ${ }^{70}$ Parasomnias are complex or bizarre behaviours that result from sleep. ${ }^{68}$ Somnambulism, sleep terror, and rapid eye movement sleep behaviour disorder (RBD) are common parasomnias that could be mistaken for nocturnal epilepsy. ${ }^{71}$ There are no literature data on the presence of these parasomnias in PNES, and studies that monitored overnight EEG video did not describe them. ${ }^{13,57}$

Popkirov et al found a comparable percentage of sleepdisordered breathing in epileptic patients and PNES patients (9\%), while periodic limb disturbance was more frequent in patients with PNES than patients with epilepsy ( $27 \%$ vs $9 \%$ ). However, the study was conducted on a limited sample (22 patients with PNES and 44 with epilepsy). ${ }^{67}$

PNES and post-traumatic stress disorder (PTSD) are often linked via experienced psychological trauma, thus sleep abnormalities, especially nightmares that occur in PTSD, might be similar in PNES. ${ }^{58}$

One theory states that the body mass index could be higher in PNES patients compared to epilepsy patients. ${ }^{72}$ As a result, sleep-related respiratory distress could be more pronounced in PNES patients than in healthy subjects. The Royal Melbourne Hospital Epilepsy Monitoring Unit is routinely using polysomnography in all patients monitored by video-EEG, and published their findings in 87 patients: 43 with epilepsy, 17 with PNES, and 4 with both. ${ }^{13}$ Data were assessed using the Pittsburgh Sleep Quality Index (PSQI), a scale evaluating sleep quality, where an interval from 0 to 5 indicates high sleep quality and above 5 low quality (PSQI) ${ }^{73}$ Patients with PNES had from the beginning higher PSQI scores (11.0) compared to patients with epilepsy (7.8). Clinically significant sleep disordered breathing (measured by Apnoea/hypopnoea index over 15) was diagnosed in 19 of $87(22 \%)$ patients. OSA (obstructive sleep apnea) was diagnosed in 15 of them, one patient was diagnosed with 
CSA (central sleep apnea), and three patients were diagnosed with mixed sleep apnea. Sleep-disordered breathing was diagnosed in 5 of 17 patients with PNES compared to 9 of 43 patients with epilepsy and 5 of 23 patients in the control group. Although the frequency of sleep-disordered breathing in patients with PNES was higher, it did not reach significance due to the limited number of subjects. The authors found significantly higher PSQI scores in PNES patients compared to epilepsy patients and control group, which is in agreement with previous findings. ${ }^{13}$ Contrary to the previously theorized relationship of BMI and PNES, quality of sleep in patients with PNES compared with epilepsy group does not seem to be associated with differences in BMI in this study.

In summary, the current literature does not describe significantly higher proportions of sleep disorders other than insomnia. However, there exists some scarce evidence of higher incidence of periodic limb movement syndrome in those patients. Therefore, further research with a higher number of patients is needed to confirm these findings further.

\section{Conclusion}

Dissociative seizures (PNES) present a relatively common and disabling disorder. There is a lack of research in its aetiology and treatment. This is probably due to the relatively low effectiveness of treatment of these patients in the past. In the last decade, however, neuropsychiatric approaches have improved diagnostics with video EEG monitoring, and randomized controlled trials have demonstrated the effectiveness of cognitive behavioural therapy.

Studies included in this review put forward that sleep problems are common in PNES patients. Besides the nature of sleep disorders, the patient may be more prone to somatization, which could be interpreted as other forms of conversion, and many PNES patients have other comorbid functional disorders. $^{7}$

Understanding the specific sleep problem of PNES, especially when sleep is essential for good health, can offer insight into improving the quality of life. Future research should utilize video EEG monitoring on a broader selection of patients with PNES to evaluate the physiology of sleep in patients with PNES, as insight into sleep disturbances may allow further understanding of the aetiology and symptoms of PNES.

In summary, contemporary literature and original research papers found more evidence of subjective sleep quality than objective, measurable changes and disorders in PNES patients. Education of patients with PNES about sleep hygiene and the significance of proper sleep habits should be included in the basic management of PNES patient. Physicians should also assess sleep in PNES patients and treat sleep disorders according to guidelines to improve overall health and quality of life.

\section{Disclosure}

The authors report no conflicts of interest for this work. We declare that we have read the journal's position on ethical publications and confirm that this text is in accordance with these guidelines.

\section{References}

1. American Psychiatric Association. Diagnostic and Statistical Manual of Mental Disorders. 5th ed ed. Arlington, VA: American Psychiatric Association; 2013.

2. LaFrance WC, Devinsky O. The treatment of nonepileptic seizures: historical perspectives and future directions. Epilepsia. 2004;45 (Suppl 2):15-21. doi:10.1111/j.0013-9580.2004.452002.x

3. Brown RJ, Bouska JF, Frow A, et al. Emotional dysregulation, alexithymia, and attachment in psychogenic nonepileptic seizures. Epilepsy Behav. 2013;29:178-183. doi:10.1016/j.yebeh.2013.07.019

4. Cerasa A, Labate A. The meaning of anxiety in patients with PNES. Epilepsy Behav. 2018;87:248. doi:10.1016/j.yebeh.2018.07.012.

5. Devinsky O, Gazzola D, LaFrance WC Jr. Differentiating between nonepileptic and epileptic seizures. Nat Rev Neurol. 2011;7:210-220. doi:10.1038/nrneurol.2011.24

6. Kuyk J, Spinhoven P, Boas WVE, van Dyck R. Dissociation in temporal lobe epilepsy and pseudo-epileptic seizure patients. J Nerv Ment Dis. 1999;187:713-720. doi:10.1097/00005053-199912000-00002

7. LaFrance WC Jr, Baker GA, Duncan R, Goldstein LH, Reuber M. Minimum requirements for the diagnosis of psychogenic nonepileptic seizures: a staged approach: a report from the international league against epilepsy nonepileptic seizures task force. Epilepsia. 2013;54 (11):2005-2018. doi:10.1111/epi.12356

8. Kanemoto K, LaFrance WC Jr, Duncan R. PNES around the world: where we are now and how we can close the diagnosis and treatment gaps-an ILAE PNES Task Force report. Epilepsia Open. 2017;2 (3):307-316. doi:10.1002/epi4.12060

9. King DW, Gallagher BB, Murvin AJ, et al. Pseudoseizures: diagnostic evaluation. Neurology. 1982;32:18-23. doi:10.1212/WNL.32.1.18

10. Haykal MA, Smith BA. Therapeutic approach to psychogenic nonepileptic seizures. Curr Treat Options Neurol. 2015;17(9):371. doi:10.1007/s11940-015-0371-4

11. Desai BT, Porter RJ, Penry JK. Psychogenic seizures: a study of 42 attacks in six patients, with intensive monitoring. Arch Neurol. 1982;39:202-209. doi:10.1001/archneur.1982.00510160008002

12. Krumholz A, Niedermeyer E. Psychogenic seizures: a clinical study with follow-up data. Neurology. 1993;33:498-502. doi:10.1212/ WNL.33.4.498

13. Phillips MCL, Costello CA, White EJ, et al. Routine polysomnography in an epilepsy monitoring unit. Epilepsy Res. 2013;105 (3):401-404. doi:10.1016/j.eplepsyres.2013.02.015

14. Sanabria-Castro A, Henríquez-Varela F, Monge-Bonilla C, LaraMaier S, Sittenfeld-Appel M. Paroxysmal events during prolonged video-video electroencephalography monitoring in refractory epilepsy. Neurologia. 2019;34(4):234-240. doi:10.1016/j. nrl.2016.12.003 
15. D'Alessio L, Giagante B, Oddo S, et al. Psychiatric disorders in patients with psychogenic nonepileptic seizures, with and without comorbid epilepsy. Seizure. 2006;15(5):333-339. doi:10.1016/j. seizure.2006.04.003

16. Shen W, Bowman ES, Markand ON. Presenting the diagnosis of pseudoseizure. Neurology. 1990;40:756-759. doi:10.1212/ WNL.40.5.756

17. Asadi-Pooya AA, Homayoun M. Psychogenic nonepileptic seizures: the sex ratio trajectory across the lifespan. Seizure. 2020;75:63-65. doi:10.1016/j.seizure.2019.12.017

18. Krawetz P, Fleisher W, Pillay N, Staley D, Arnett J, Maher J. Family functioning in subjects with pseudoseizures and epilepsy. J Nerv Ment Dis. 2001;189:38-43. doi:10.1097/00005053-200101000-00007

19. Jennum P, Ibsen R, Kjellberg J. Welfare consequences for people diagnosed with nonepileptic seizures: a matched nationwide study in Denmark. Epilepsy Behav. 2019;98(Pt A):59-65. doi:10.1016/j. yebeh.2019.06.024

20. Reuber M, Pukrop R, Bauer J, Helmstaedter C, Tessendorf N, Elger CE. Outcome in psychogenic nonepileptic seizures: 1 to 10-year follow-up in 164 patients. Ann Neurol. 2003;53:305-311. doi:10.1002/ana.3000

21. de Timary P, Fouchet P, Sylin M, et al. Nonepileptic seizures: delayed diagnosis in patients presenting with electroencephalographic (EEG) or clinical signs of epileptic seizures. Seizure. 2002;11:193-197. doi:10.1053/seiz.2001.0617

22. Asadi-Pooya AA, Tinker J. Delay in diagnosis of psychogenic nonepileptic seizures in adults: a post hoc study. Epilepsy Behav. 2017;75:143-145. doi:10.1016/j.yebeh.2017.08.005

23. Selwa LM, Geyer J, Nikakhtar N, Brown MB, Schuh LA, Drury I. Nonepileptic seizure outcome varies by type of spell and duration of illness. Epilepsia. 2000;41:1330-1334. doi:10.1111/j.15281157.2000.tb04613.x

24. Bajestan SN, LaFrance WC Jr. Clinical approaches to psychogenic nonepileptic seizures. Focus. 2016;14(4):422-431. doi:10.1176/appi. focus. 20160020

25. Bazil CW, Legros B, Kenny E. Sleep structure in patients with psychogenic nonepileptic seizures. Epilepsy Behav. 2003;4 (4):395-398. doi:10.1016/S1525-5050(03)00120-3

26. Uliaszek AA, Prensky E, Baslet G. Emotion regulation profiles in psychogenic nonepileptic seizures. Epilepsy Behav. 2012;23:364-369. doi:10.1016/j.yebeh.2012.01.009

27. Guillen A, Curot J, Birmes PJ, et al. Suicidal ideation and traumatic exposure should not be neglected in epileptic patients: a multidimensional comparison of the psychiatric profile of patients suffering from epilepsy and patients suffering from psychogenic nonepileptic seizures. Front Psychiatry. 2019;10:303. doi:10.3389/ fpsyt.2019.00303

28. Bowman ES, Markand ON. Psychodynamics and psychiatric diagnoses of pseudoseizure subjects. Am J Psychiatry. 1996;153:57-63.

29. Asadi-Pooya AA, Bahrami Z. Sexual abuse and psychogenic nonepileptic seizures. Neurol Sci. 2019;40(8):1607-1610. doi:10.1007/ s10072-019-03887-3

30. Martino I, Bruni A, Labate A, et al. Psychopathological constellation in patients with PNES: a new hypothesis. Epilepsy Behav. 2018;78:297-301. doi:10.1016/j.yebeh.2017.09.025

31. Baslet G, Roiko A, Prensky E. Heterogeneity in psychogenic nonepileptic seizures: understanding the role of psychiatric and neurological factors. Epilepsy Behav. 2010;17:236-241. doi:10.1016/j. yebeh.2009.12.008

32. Van der Kruijs SJ, Bodde NM, Aldenkamp AP. Psychophysiological biomarkers of dissociation in psychogenic nonepileptic seizures. Acta Neurol Belg. 2011;111(2):99-103.

33. Bakvis P, Roelofs K, Kuyk J, Edelbroek PM, Swinkels WA, Spinhoven P. Trauma, stress, and preconscious threat processing in patients with psychogenic nonepileptic seizures. Epilepsia. 2009;50:1001-1011. doi:10.1111/j.1528-1167.2008.01862.x
34. Urbanek M, Harvey M, McGowan J, Agrawal N. Regulation of emotions in psychogenic nonepileptic seizures. Epilepsy Behav. 2014;37:110-115. doi:10.1016/j.yebeh.2014.06.004

35. Roberts NA, Burleson MH, Torres DL, et al. Emotional reactivity as a vulnerability for psychogenic nonepileptic seizures? Responses while reliving specific emotions. $J$ Neuropsychiatry Clin Neurosci. 2020;32(1):95-100. doi:10.1176/appi.neuropsych.19040084

36. Goldstein LH, Mellers JD. Ictal symptoms of anxiety, avoidance behaviour, and dissociation in patients with dissociative seizures. J Neurol Neurosurg Psychiatry. 2006;77:616-621. doi:10.1136/ jnnp.2005.066878

37. Williams IA, Levita L, Reuber M. Emotion dysregulation in patients with psychogenic nonepileptic seizures: a systematic review based on the extended process model. Epilepsy Behav. 2018;86:37-48. doi:10.1016/j.yebeh.2018.06.049

38. Quinn M, Schofield M. Middleton W: conceptualization and treatment of psychogenic nonepileptic seizures. J Trauma Dissociation. 2008;9:63-84. doi:10.1080/15299730802073676

39. Vasta R, Cerasa A, Sarica A, et al. The application of artificial intelligence to understand the pathophysiological basis of psychogenic nonepileptic seizures. Epilepsy Behav. 2018;87:167-172. doi:10.1016/j.yebeh.2018.09.008

40. Kalogjera-Sackellares D Psychological Disturbances in Patients with Pseudoseizures; in Psychological Disturbances in Epilepsy. Edited by Sackellares JC, Berent $\mathrm{S}$ Oxford, England, Butterworth Heinemann, 1996.

41. Duncan R, Oto M. Predictors of antecedent factors in psychogenic nonepileptic attacks: multivariate analysis. Neurology. 2008;71:1000-1005. doi:10.1212/01.wnl.0000326593.50863.21

42. Myers L, Perrine K, Lancman M, Fleming M, Lancman M. Psychological trauma in patients with psychogenic nonepileptic seizures: trauma characteristics and those who develop PTSD. Epilepsy Behav. 2013;28:121-126. doi:10.1016/j.yebeh.2013. 03.033

43. Van der Hart O, Nijenhuis E, Steele K, Brown D. Trauma-related dissociation: conceptual clarity lost and found. Aust N Z J Psychiatry. 2004;38:906-914. doi:10.1080/j.1440-1614.2004.01480.x

44. LaFrance WC, Wincze JP. Treating Nonepileptic Seizures: Therapist Guide. New York: Oxford University Press; 2015.

45. Dixit R, Popescu A, Bagić A, Ghearing G, Hendrickson R. Medical comorbidities in patients with psychogenic nonepileptic spells (PNES) referred for video-EEG monitoring. Epilepsy Behav. 2013;28:137-140. doi:10.1016/j.yebeh.2013.05.004

46. Asmussen SB, Kirlin KA, Gale SD, Chung SS. Differences in self-reported depressive symptoms between patients with epileptic and psychogenic nonepileptic seizures. Seizure. 2009;18:564-566. doi:10.1016/j.seizure.2009.05.006

47. LaFrance WC Jr, Miller IW, Ryan CE. Cognitive behavioral therapy for psychogenic nonepileptic seizures. Epilepsy Behav. 2009;14 (4):591-596. doi:10.1016/j.yebeh.2009.02.016

48. Kamil SH, Qureshi M, Patel RS. Cognitive behavioral therapy (CBT) in psychogenic nonepileptic seizures (PNES): A case report and literature review. Behav Sci (Basel). 2019;9(2):E15. doi:10.3390/ bs9020015

49. Ferrari R. Writing narrative style literature reviews. Med Writing. 2015;24(4):230-235. doi:10.1179/2047480615Z.000000000329

50. Latreille V, Dworetzky BA, Baslet G, Pavlova M. Sleep disturbances in patients with psychogenic nonepileptic seizures: is it all subjective? A prospective pilot study of sleep-wake patterns. Seizure. 2019;65:124-128. doi:10.1016/j.seizure.2019.01.016

51. Latreille V, Baslet G, Sarkis R, Pavlova M, Dworetzky BA. Sleep in psychogenic nonepileptic seizures: time to raise a red flag. Epilepsy Behav. 2018;86:6-8. doi:10.1016/j.yebeh.2018.07.001

52. Thacker K, Devinsky O, Perrine K, Alper K, Luciano D. Nonepileptic seizures during apparent sleep. Ann Neurol. 1993;33:414-418. doi:10.1002/ana.410330417 
53. Smith D, Defalla BA, Chadwick DW. The misdiagnosis of epilepsy and the management of refractory epilepsy in a specialist clinic. QJM. 1999;92:15-23. doi:10.1093/qjmed/92.1.15

54. Reuber M. Psychogenic nonepileptic seizures: answers and questions. Epilepsy Behav. 2008;12:622-635. doi:10.1016/j.yebeh.2007.11.006

55. Bazil CW, Walczak TS. Effects of sleep and sleep stage on epileptic and nonepileptic seizures. Epilepsia. 1997;38:56-62. doi:10.1111/ j.1528-1157.1997.tb01077.x

56. Duncan R, Oto M, Russell AJ, Conway P. Pseudosleep events in patients with psychogenic nonepileptic seizures: prevalence and associations. $J$ Neurol Neurosurg Psychiatry. 2004;75 (7):1009-1012. doi:10.1136/jnnp.2003.022632

57. Nežádal T, Hovorka J, Herman E, Němcová I, Bajaček $M$, Stichová E. Psychogenic non-epileptic seizures: our video-EEG experience. Neurol Res. 2011;33(7):694-700. doi:10.1179/ 1743132811Y.0000000003

58. Pavlova MK, Allen RM, Dworetzky BA. Sleep in psychogenic nonepileptic seizures and related disorders. Clin EEG Neurosci. 2015;46 (1):34-41. doi:10.1177/1550059414560565

59. Orbach D, Ritaccio A, Devinsky O. Psychogenic, nonepileptic seizures associated with video-EEG-verified sleep. Epilepsia. 2003;44 (1):64-68. doi:10.1046/j.1528-1157.2003.29302.x

60. Seneviratne U, Minato E, Paul E. Seizures by the clock: temporal patterns of psychogenic nonepileptic seizures. Epilepsy Behav. 2017;76:71-75. doi:10.1016/j.yebeh.2017.08.025

61. Kapur J, Pillai A, Henry TR. Psychogenic elaboration of simple partial seizures. Epilepsia. 1995;36:1126-1130. doi:10.1111/j.15281157.1995.tb00471.x

62. Graham CD, Kyle SD. A preliminary investigation of sleep quality in functional neurological disorders: poor sleep appears common, and is associated with functional impairment. $J$ Neurol Sci. 2017;378:163-166. doi:10.1016/j.jns.2017.05.021

63. Cramer JA, Perrine K, Devinsky O, Meador K. A brief questionnaire to screen for quality of life in epilepsy: the QOLIE-10. Epilepsia. 1996;37(6):577-582. doi:10.1111/j.1528-1157.1996.tb00612.x
64. Jones B, Reuber M, Norman P. Correlates of health-related quality of life in adults with psychogenic nonepileptic seizures: a systematic review. Epilepsia. 2016;57(2):171-181. doi:10.1111/epi.13268

65. Sylvia LG, Chang WC, Kamali M, et al. Sleep disturbance may impact treatment outcome in bipolar disorder: a preliminary investigation in the context of a large comparative effectiveness trial. J Affect Disord. 2018;225:563-568. doi:10.1016/j.jad.2017.08.056

66. Bazil CW, Castro LHM, Walczak TS. Diurnal and nocturnal seizures reduce REM in patients with temporal lobe epilepsy. Arch Neurol. 2000;57:363-368. doi:10.1001/archneur.57.3.363

67. Popkirov S, Stone J, Derry CP. Abnormal sleep in patients with epileptic or dissociative (nonepileptic) seizures: a polysomnography study. Eur J Neurol. 2019;26(2):255-260. doi:10.1111/ene.13798

68. Schenck CH, Mahowald MW. Parasomnias. Managing bizarre sleep-related behavior disorders. Postgrad Med J. 2000;107:145-156. doi:10.3810/pgm.2000.03.937

69. Mellers JD. The approach to patients with "nonepileptic seizures. Postgrad Med J. 2005;81:498-504. doi:10.1136/pgmj.2004.029785

70. Swick TJ. Treatment paradigms for cataplexy in narcolepsy: past, present, and future. Nat Sci Sleep. 2015;7:159-169. doi:10.2147/ NSS.S92140

71. Wills L, Garcia J. Parasomnias: epidemiology and management. CNS Drugs. 2002;16:803-810. doi:10.2165/00023210-200216120-00002

72. Marquez AV, Farias ST, Apperson M, et al. Psychogenic nonepileptic seizures are associated with an increased risk of obesity. Epilepsy Behav. 2004;5(1):88-93. doi:10.1016/j.yebeh.2003.10.019

73. Buysse DJ, Reynolds CF 3rd, Monk TH, Berman SR, Kupfer DJ. The pittsburgh sleep quality index: a new instrument for psychiatric practice and research. Psychiatry Res. 1989;28(2):193-213. doi:10.1016/0165-1781(89)90047-4

\section{Publish your work in this journal}

Nature and Science of Sleep is an international, peer-reviewed, open access journal covering all aspects of sleep science and sleep medicine, including the neurophysiology and functions of sleep, the genetics of sleep, sleep and society, biological rhythms, dreaming, sleep disorders and therapy, and strategies to optimize healthy sleep.
The manuscript management system is completely online and includes a very quick and fair peer-review system, which is all easy to use. Visit http://www.dovepress.com/testimonials.php to read real quotes from published authors. 